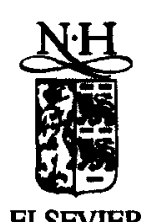

Journal of Luminescence 64 (1995) 75-79

\title{
Temperature-annealing-cycling hole spectra of dye and dye-oligonucleotide doped in polymer films
}

\author{
Ta-Chau Chang ${ }^{a, *}$, Chien-Chih Chiang ${ }^{b}$, Shiow-Hwa Chou ${ }^{a}$, Konan Peck ${ }^{c}$ \\ ${ }^{3}$ Institute of Atomic and Molecular Sciences, Academia Sinica, P.O. Box 23-166, Taipei 10764, Taiwan \\ 'Department of Chemistry, National Taiwan University, Taipei 10764, Taiwan \\ 'Institute of Biomedical Sciences, Academia Sinica, Taipei, 11529, Taiwan
}

Received 24 August 1994; revised 8 November 1994; accepted 12 December 1994

\begin{abstract}
Temperature-annealing-cycling hole spectra are introduced to examine the hole burning kinetics of dye and dyeoligonucleotide in polymer films. When a chromophore binds to a large group of oligonucleotide, the burning efficiency decreases and the electron-phonon coupling strength increases.
\end{abstract}

\section{Introduction}

Nonphotochemical hole-burning (NPHB) spectroscopy of chromophores in amorphous glasses has been extensively applied to investigate the chromophore-glass interaction at low temperature [1-3]. Recently, Chang and co-workers [4] have demonstrated that temperature-annealing-cycling (TAC) hole spectra can provide the information on electron-phonon coupling and the distribution of Stokes energy shift. The TAC hole spectra are taken as follows: a hole-burned (HB) spectrum is obtained at burning temperature $\left(T_{\mathrm{b}}\right)$, after a hole is produced. Then the temperature is raised to the annealing temperature $\left(T_{\mathrm{a}}\right)$, the annealing spectrum is taken at $T_{\mathrm{a}}$. Furthermore, the temperature is cooled back to the cycling temperature $\left(T_{\mathrm{c}}\right)$, the cycling spectrum is recorded at $T_{\mathrm{c}}$. It is noted that

\footnotetext{
* Corresponding author.
}

$T_{\mathrm{c}}=T_{\mathrm{b}}$. They have found that the integrated hole area is reduced while raising the temperature to $T_{\mathrm{a}}$. However, part of the holes is recovered when the system is cooled back to the temperature at $T_{\mathrm{c}}$, where $T_{\mathrm{c}}=T_{\mathrm{b}}$. The irreversible part of the hole filling at high temperature results from the structural relaxation [5]. The reversible change of the hole area is attributed to linear electron-phonon coupling [4-7]. In addition, they proposed that the difference of hole area between annealing and cycling spectra provides the distribution of Stokes energy shift [4].

The purpose of this work is to examine how chemical binding of a chromophore to a large group of oligonucleotide of guanosine affects hole burning kinetics. We have studied TAC hole spectra of 5-(and 6-) carboxy-X-rhodamine N-hydroxy succinimidyl ester (RhSE) and the RhSE molecule with binding to an oligonucleotide of ten bases of guanosine ( $\mathrm{RhSE}-10 \mathrm{G})$ doped in polyvinyl 
alcohol (PVOH) films. It has been found that the burning efficiency in RhSE-10G/PVOH is lower than that in RhSE/PVOH. In addition, our results suggest that the strength of electron-phonon coupling is larger in the system of RhSE-10G/PVOH than that of RhSE/PVOH.

\section{Experimental}

The experimental setup and the method for sample preparation have been described elsewhere $[4,8]$. Hole was burned by using $\mathrm{Ar}^{+}$laser pumping a ring dye laser (Coherent 899-01) with a width of $\sim 1 \mathrm{~cm}^{-1}$ and the photon flux of $\leq 40 \mathrm{~mW} / \mathrm{cm}^{2}$ for several minutes. The spot size of the burning beam was $\sim 1 \mathrm{~mm}$ and that of the reading beam was $\sim 200 \mu \mathrm{m}$. Extreme care was taken to ensure that the cross-section of the probe beam at the sample should be inside the area of the burn laser. The absorption spectra were obtained by using a home-made double beam spectrometer with the resolution of $\sim 0.3 \AA$. The samples were mounted on a brass plate with several drilled holes of $\sim 1 \mathrm{~mm}$ diameter, which were put into a Janis dewer and were cooled by passing cold helium gas over it. The deviation of the temperature is $\pm 0.5 \mathrm{~K}$ at low temperature. All spectra were taken after the temperature stabilized for $\geq 30$ min because of the low thermal conductivity of polymer samples.

\section{Results and discussion}

Fig. 1 shows typical TAC spectra of RhSE/ PVOH and RhSE-10G/PVOH. The burning times are 3 and $10 \mathrm{~min}$ for RhSE/PVOH and RhSE10G/PVOH with a flux of $\sim 12 \mathrm{~mW} / \mathrm{cm}^{2}$ at $\lambda_{\mathrm{B}} \approx 5812 \AA$, respectively. A sharp $\mathrm{ZPH}$ accompanied by an asymmetric pseudophonon side band hole (PSBH) with tailing on the low energy side is observed in both HB spectra. In addition, a shallow hole located at $\sim 3 \mathrm{~nm}$ to the red of the $\mathrm{ZPH}$ is observed in Fig. 1. The dotted lines present the fit to the experimental data. The details of the simulation approach can be found elsewhere [8]. In brief, the method consists of separately calculating three Gaussian line shapes including a ZPH, a PSBH,

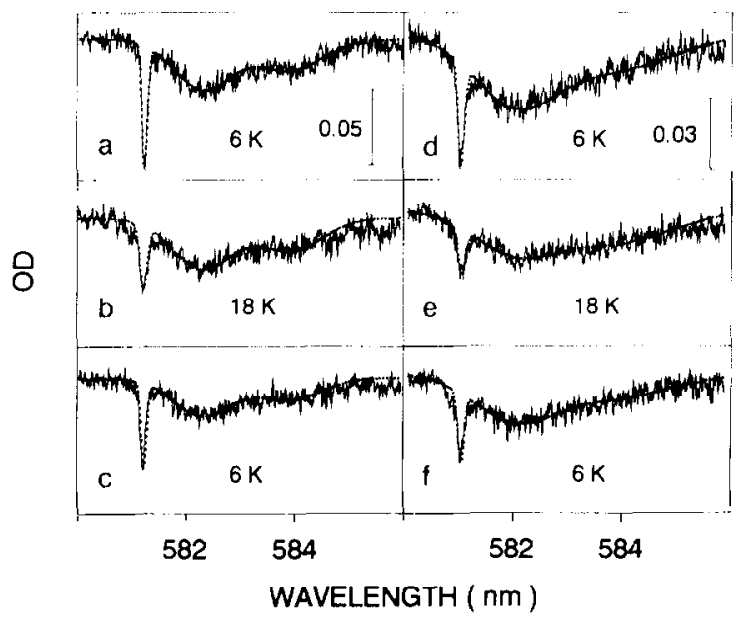

Fig. 1. Typical temperature-annealing-cycling hole spectra of RhSE/PVOH (a)-(c) and RhSE-10G/PVOH (d)-(f). The dotted lines present the fit to the experimental data by using the widths of a $\mathrm{ZPH}$, a PSBH, and a shallow hole as $(0.6,6.5,7) \AA$ for

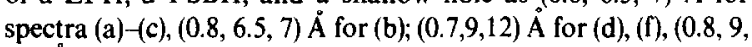
12) $\AA$ for (e).

and a shallow hole. It is found that the width of $\mathrm{ZPH}$ in the cycling spectra is approximately identical to that in the HB spectra. However, the width of $\mathrm{ZPH}$ in the annealing spectra is slightly broader at higher temperatures for both systems. Unfortunately, we are unable to determine the homogeneous line width in our present investigation. Nevertheless, the reversible hole width in the TAC spectra implies that the spectral diffusion is unlikely to be the dominant hole broadening mechanism because the local structure randomized by the spectral diffusion is an irreversible process [9].

In addition, Fig. 1 shows that the burning efficiency of RhSE-10G/PVOH is less than that of $\mathrm{RhSE} / \mathrm{PVOH}$. It is noted that the peak center of the low energy absorption band is shifted from $\sim 574 \mathrm{~nm}$ in $\mathrm{RhSE} / \mathrm{PVOH}$ to $\sim 580 \mathrm{~nm}$ in RhSE$10 \mathrm{G} / \mathrm{PVOH}$. The optical density of RhSE$10 \mathrm{G} / \mathrm{PVOH}$ is approximately twice stronger than that of RhSE/PVOH at $5812 \AA$ [4]. However, even under the high fluence condition the hole depth of $\mathrm{ZPH}$ in the spectra of RhSE/PVOH is more than twice that of $\mathrm{RhSE}-10 \mathrm{G} / \mathrm{PVOH}$. Lower burning efficiency in RhSE-10G/PVOH suggests that the oligonucleotide binding to the RhSE molecule is involved in the processes of hole formation. 
Nakanishi and co-workers [10] have studied the HB spectra of nile blue doped in PVOH and crosslinked PVOH derivatives. They found that the depth of ZPH decreases as a function of the length of the cross-linker introduced to the PVOH backbone. The decrease of the hole depth is attributed to the change of the TLSs distribution around the dye entrapped in the void space induced by the crosslinking. In this work, the large size of oligonucleotide could enlarge the void around the chromophore in the polymer matrix. As a result, the effective density of TLSs around the chromophore can be reduced upon the optical excitation.

Furthermore, Fig. 1 presents that the integrated hole area is reduced while raising the temperature to $T_{\mathrm{a}}$. However, part of the holes is recovered when the system is cooled back to the burning temperature at $T_{\mathrm{c}}$, where $T_{\mathrm{c}}=T_{\mathrm{b}}$. Possible mechanisms for the hole filling involve spontaneous filling and thermally activated processes. We have measured the integrated hole area of ZPH as a function of waiting time to examine the spontaneous hole filling. Fig. 2 shows the plots of hole area as a function

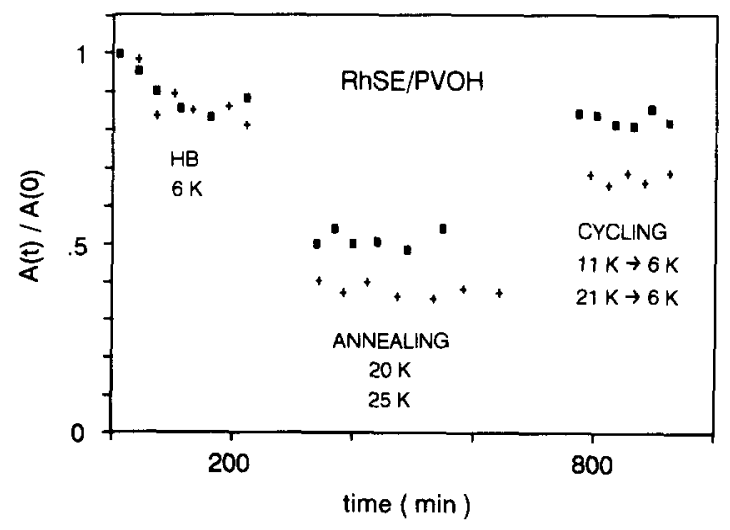

Fig. 2. The plots of the hole areas as a function of waiting time under the burning temperature condition $(\square,+)$ for the first $3.5 \mathrm{~h}$, and the annealing temperature condition $(\square,+)$ for the last $5 \mathrm{~h}$, and the cycling temperature condition $(\square,+)$. In the burning condition, two set of data $(\square,+)$ are obtained at $T_{\mathrm{b}}=6 \mathrm{~K}$. The annealing spectra are measured right after the HB spectra, respectively, where: $\square$ : raise sample to $T_{\mathrm{a}}=20 \mathrm{~K}$ and read at $20 \mathrm{~K} ;+$ : raise sample to $T_{\mathrm{n}}=25 \mathrm{~K}$ and read at $25 \mathrm{~K}$. However, the cycling spectra are obtained independently, where: $\square$ : cools sample from $T_{\mathrm{a}}=11 \mathrm{~K}$ to $T_{\mathrm{c}}=T_{\mathrm{b}}=6 \mathrm{~K}$ and read at $6 \mathrm{~K} ;+:$ cool sample from $T_{\mathrm{a}}=21 \mathrm{~K}$ to $T_{\mathrm{c}}=T_{\mathrm{b}}=6 \mathrm{~K}$ and read at $6 \mathrm{~K}$. of waiting time for RhSE/PVOH obtained under various conditions. Under the burning temperature conditions at $T_{\mathrm{b}}=6 \mathrm{~K}$, the $\mathrm{ZPH}$ is reduced by $\sim 15 \%$ of hole area in the first hour, and then the reduction of the hole area is insignificant. Under the annealing temperature condition, the integrated areas of ZPHs are nearly identical within experimental error for four hours waiting time. Under the cycling temperature condition, the areas of ZPHs are also approximately identical for more than three hours waiting time. It indicates that the change of the hole area in the TAC spectra is mainly attributed to the thermally activated hole filling process.

In our previous work [4], we have demonstrated that the difference of hole reduction between annealing and cycling spectra can be described by the linear electron-phonon coupling. The theory of the linear electron-phonon coupling suggests that the integrated intensity of the ZPH is temperature-dependent because of the spectral redistribution of the thermal occupation of the phonon state $[2,11]$. Therefore, the ratio of $I_{\mathrm{ZPH}}\left(T_{\mathrm{a}}\right) / I_{\mathrm{ZPH}}\left(T_{\mathrm{c}}\right)$ as a function of temperature should follow the relationship of [4]

$$
I_{\mathrm{ZPH}}\left(T_{\mathrm{a}}\right) / I_{\mathrm{ZPH}}\left(T_{\mathrm{c}}\right)=\exp \left[-2 S_{0} \tilde{n}\left(T_{\mathrm{a}}\right)\right]
$$

while $T_{\mathrm{c}} \rightarrow 0 . S_{0}$ is the coupling constant at $T=0 \mathrm{~K}$ and $\tilde{n}(T)$ is the thermal occupation numbers at mean phonon frequency of $\omega_{\mathrm{p}}, \tilde{n}(T)=[\exp$ $\left.\left(\hbar \omega_{\mathrm{p}} / k_{\mathrm{B}} T\right)-1\right]^{-1}$. The experimental results of the counterpart of $I_{\mathrm{ZPH}}\left(T_{\mathrm{a}}\right) / I_{\mathrm{ZPH}}\left(T_{\mathrm{c}}\right)$ for both systems are shown in Fig. 3. The solid curves in Fig. 3 represent the numerical fitting of Eq. (1) to the experimental data. The magnitude of $\omega_{\mathrm{p}} \approx 28 \mathrm{~cm}^{-1}$ inferred from the frequency difference between ZPH and PSBH shows no significant difference between these two systems. We further determine the electron-phonon coupling parameter by varying the magnitude of $S_{0}$ to simulate our experimental results. It is found that the magnitude of $S_{0}$ is $\sim 0.7$ for $\mathrm{RhSE} / \mathrm{PVOH}$ and $\sim 1.0$ for RhSE-10G/PVOH. The agreement between the fits and our experimental data indicates that the difference of hole area between annealing and cycling spectra is attributed to the linear electronphonon coupling. Our results suggest that the 


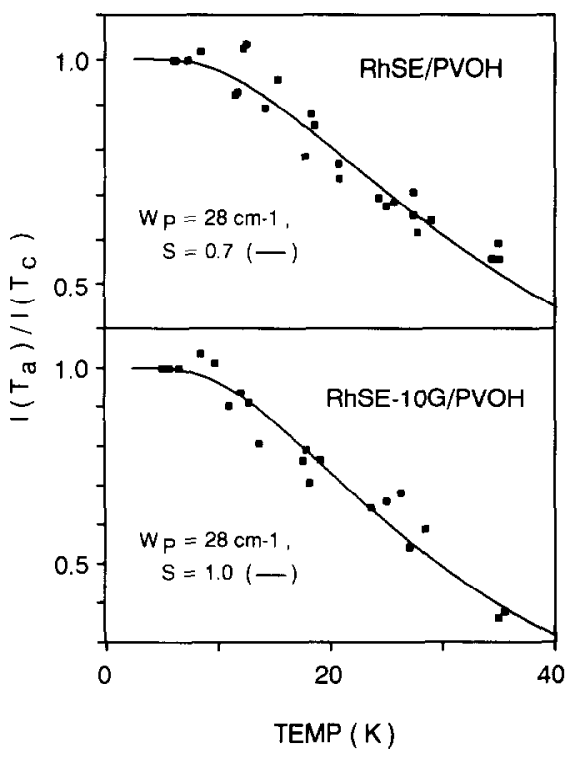

Fig. 3. The plots of the ratio of $I_{\mathrm{ZPH}}\left(T_{\mathrm{a}}\right) / I_{\mathrm{ZPH}}\left(T_{\mathrm{c}}\right)$ as a function of annealing temperature for (a) RhSE/PVOH and (b) RhSE$10 \mathrm{G} / \mathrm{PVOH}$. The solid lines are the fit of Eq. (1) to the experimental data.

electron-phonon coupling strength of RhSE$10 \mathrm{G} / \mathrm{PVOH}$ is larger than that of RhSE/PVOH.

Saiken and co-workers [12] have found that in the systems of rhodamine-640 doped in different polymers the electron-phonon coupling is stronger if the side group on the polymer chain is larger and stiffer. They proposed that the electron-phonon coupling depends upon the size of the free volume around the chromophore in the polymers. In our work, the large oligonucleotide binding to the chromophore may provide larger free volume around the chromophore in the polymer matrices. The larger the free volume in the polymer provides more freedom for the guest molecule. It could optimize the coupling strength. Further examination on the free volume behavior will be performed by measuring the dephasing of these two systems. The larger the free volume is, the faster dephasing should be observed [13].

Other possible mechanisms involved for the larger $S_{0}$ in the system of RhSE-10G/PVOH are the dipole-dipole interaction and additional phonon coupling modes. The linear electron-phonon coupling of $S_{0}$ is given by $S_{0}=\Sigma_{i} S_{i}=\Sigma_{i} m \omega_{i} q_{i}^{2} / 2 \hbar$, where $q_{i}$ is the displacement of the equilibrium position upon a phototransition. The larger magnitude of $S_{0}$ in RhSE-10G/PVOH could imply that a larger displacement between ground and excited states is involved. The larger displacement may be attributed to a larger change of dipole moment upon the optical excitation. If RhSE is on a base stacked configuration, this could introduce chargetransfer character into $S_{1}$ [14]. Consequently, a larger change on dipole moment is produced and a stronger electron-phonon coupling behavior is presented in the spectra of RhSE-10G/PVOH. Unfortunately, the structural configuration of RhSE$10 \mathrm{G}$ complex is not clear at this moment.

Since $S_{0}$ consists of all the coupling between the phonon modes and the excited chromophore, additional phonon modes due to the ten bases of guanosine may also enlarge the magnitude of $S_{0}$. It is known that the phonon side band is due to the coupling of phonon modes. Fig. 1 shows that the shape of the PSBH in RhSE-10G/PVOH is broader than that in $\mathrm{RhSE} / \mathrm{PVOH}$. In addition, we have proposed that the difference of hole area between annealing and cycling spectra can provide the phonon distribution of coupling modes [4]. Our results show that the difference between annealing and cycling spectra of RhSE-10G/PVOH is larger than that of $\mathrm{RhSE} / \mathrm{PVOH}$ in the temperature regime of $10-17 \mathrm{~K}$. It is suggested that additional lower phonon modes are coupled to the excited chromophore.

\section{Conclusions}

In summary, we have applied temperature-annealing-cycling hole spectra to investigate how the large group of oligonucleotide binding to a chromophore affects the hole-burning kinetics. It is found that binding an oligonucleotide to a chromophore decreases the hole-burning efficiency, but increases the electron-phonon coupling strength. The former phenomenon can be described by the reduction of the effective density of TISs around the RhSE due to the larger free volume created via the larger size of oligonucleotide. The latter feature is temporarily described by three proposed mechanisms. The larger free volume in the polymer matrix 
allows the guest molecule to move for optimizing the electron-phonon coupling. The stacked configuration of RhSE-10G may enhance the dipoledipole interaction. Additional lower phonon modes due to the oligonucleotide may couple to the excited chromophore to enlarge the electron-phonon coupling. Further examination on these mechanisms is in progress.

\section{Acknowledgements}

This work was supported by Academia Sinica and the NSC of the Republic of China (Grant NO. NSC-83-0208-M-001-091). T.C.C. acknowledges Professor G.J. Small at Iowa State University for his valuable discussion.

\section{References}

[1] G.J. Small, in: Spectroscopy and Excitation Dynamics of Condensed Molecular Systems, eds. V.M. Agranovich, R.M. Hochstrasser (North-Holland, Amsterdam, 1983).
[2] J. Friedrich, D. Haarer, Angew. Chemie 96 (1984) 96; Int. Ed. Engl. 23 (1984) 113.

[3] W.E. Moerner, Topics in Current Physics, Persistent Spectral Hole Burning: Science and Applications (SpringerVerlag, New York, 1988).

[4] T.-C. Chang, C.C. Chiang, G.J. Small, S.H. Chou, Chem, Phys. Lett. 223 (1994) 190.

[5] W. Köhler, J. Meiler, J. Friedrich, Phys. Rev. B 35 (1987) 4031.

[6] A. Furusawa, K. Horie, J. Chem. Phys. 94 (1991) 80.

[7] B. Sauter, Th. Basche, C. Brauchle, J. Opt. Soc. Am. B 9 (1992) 804.

[8] T.-C. Chang, C.C. Chiang, S.H. Chou, K. Peck, J. Phys. Chem., in press.

[9] L.R. Narasimhan, K.A. Littau, D.W. Pack, Y.S. Bai, A. Elschner, M.D. Fayer, Chem. Rev. 90 (1990) 439.

[10] S. Nakanish, Y. Miyawaki, M. Nishikawa, M. Amano, A. Fujiwara, M. Jitou, H. Itoh, M. Kawase, J. Chem. Phys. 100 (1994) 3442.

[11] K.K. Rebane, Impurity Spectra of Solid (Plenum, New York, 1970).

[12] S. Saikan, Y. Kanematsu, R. Shiraishi, T. Nakabayashi, T. Kushida, J. Lumin. 38 (1987) 15.

[13] H.P.H. Thijssen, S. Völker, Chem. Phys. Lett. 120 (1985) 496.

[14] G.J. Small, private communication. 